\title{
Comparison of the coherent radiation-induced microbunching instability in a free-electron laser and a magnetic chicane
}

\author{
S. Reiche and J. B. Rosenzweig \\ Department of Physics \& Astronomy, University of California, Los Angeles, California 90095-1547
}

(Received 10 February 2003; published 24 April 2003)

\begin{abstract}
A self-amplified spontaneous emission free-electron laser (SASE FEL) is a device which is based on the creation of a very intense, relativistic electron beam which has very little temperature in all three phase planes. The beam in this system is described as having "high brightness," and when it is bent repetitively in a magnetic undulator, undergoes a radiation-mediated microbunching instability. This instability can amplify the original radiation amplitude at a particular, resonant wavelength by many orders of magnitude. In order to obtain high brightness beams, it is necessary to compress them to obtain higher currents than available from the electron source. Compression is accomplished by the use of magnetic chicanes, which are quite similar to, if much longer than, a single period of the undulator. It should not be surprising that such chicanes also support a radiation-mediated microbunching interaction, which has recently been investigated, and has been termed coherent synchrotron radiation (CSR) instability. The purpose of this paper is to compare and contrast the characteristics of the closely related FEL and CSR microbunching instabilities. We show that a high-gain regime of the CSR instability exists which is formally similar to the FEL instability.
\end{abstract}

DOI: 10.1103/PhysRevSTAB.6.040702

PACS numbers: $41.60 .-\mathrm{m}$

\section{INTRODUCTION}

A linear accelerator consists mainly of nominally straight accelerating structures and focusing elements, but also often relies on sections in the beam line where the electron beam is transversely deflected. In addition to bending magnets used to connect different beam lines that have a relative offset, there are dispersive sections, such as magnetic chicanes, in which the beam is finally placed along its original axis. One of the main purposes of such a bending configuration is to manipulate the bunch length. This manipulation requires a time-energy chirp to be applied to the electron bunch by the linear accelerator, with the correlation partially or fully removed by the beam dynamics in the chicane. Such a scheme is referred to as a bunch compressor. In the process of bending the beam in the chicane, broadband synchrotron radiation is emitted. The amplitude of this radiation at wavelengths similar to, or longer than, the bunch dimension can be greatly enhanced by constructive interference. This phenomenon, which is known as coherent synchrotron radiation (CSR), can lead to significant, undesirable distortions of the beam's longitudinal and transverse phase space distributions, as well as introducing correlations between phase planes.

Similar in layout to the chicane, but much smaller in size, is a single period of an undulator or wiggler magnet. The periodic deflection of the electron beam also causes the spontaneous emission of synchrotron radiation. The advantages of undulators or wigglers, which consist of a large number of such periodic bending arrays, as radiation sources are twofold: (i) the radiation power is accumulated over the entire undulator length in a compact angular region, and (ii) the periodic nature of the radiative process narrows the bandwidth in the angular spectrum around resonant frequencies through repetitive interference effects. In addition, these resonant frequencies are tunable by simply changing the driving beam energy.

The spontaneous synchrotron radiation emitted in an undulator or wiggler can act back on the electron beam. Again, because of the repetitive nature of the interaction, the emitted radiation can modulate the beam energy on the scale of the resonant wavelength [1], by forcing the electrons to exchange energy with the field through motion transverse to the field propagation vector. The emitted radiation modulates the beam particles forward of the emitting electron, because the light travels essentially along the nominal beam line path $(s)$ at speed $c$, which is faster than the electron velocity. Because the undulator is, similar to the magnetic chicane, a dispersive device, the energy modulation is eventually transferred into a temporal (current) modulation termed microbunching. Because the coherence of the radiation at the resonant wavelength is enhanced by this microbunching, more radiation power is emitted, and this feedback mechanism can drive a collective instability [2,3], where an initial bunching and the corresponding emitted radiation is amplified up to a point where maximum bunching (saturation) is achieved. This microbunching instability typically occurs on a scale much shorter than the bunch length, and is the operating principle of the free-electron laser [4] (FEL), where the instability is intended. To enhance the performance of an FEL, bunch compressors are often incorporated in the beam line to shorten the bunch length, increasing the driving beam current and beam brightness, and thus the FEL gain.

Although it is explicitly sought in an undulator or wiggler, the interaction of the electron bunch with the coherent, spontaneous radiation within a magnetic 
chicane can degrade the electron beam quality [5], reducing the performance of a succeeding FEL employed on the compressed beam. On their curved trajectory in bend magnets, the electrons emit radiation, which again moves forward in the beam, because the radiation propagates faster, and in a straight line. The radiation field and electron cross at an angle (typically much larger than in an FEL undulator) yielding a longitudinal electric field component, which changes the electron energy [6]. The difference between this energy exchange mechanism and that in an undulator is purely semantic. In the undulator, we define longitudinal as along $z$, the undulator axis, and thus energy exchange is possible because the electron travels transverse to the nominal radiation direction, along the electric field polarization direction. In the chicane magnet, we define longitudinal to be always locally along the direction of the electron $s$, so "transverse" motion of the beam's central trajectory is forbidden by definition. Thus the field propagation vector is now defined as having a transverse component, and the electric field as having a longitudinal component, allowing energy exchange. In analogy to an undulator the dispersion causes a growth in the beam current modulation and thus in the coherence level of the synchrotron radiations [7]. We shall see that the way in which microbunching due to energy modulation asserts itself in the chicane is qualitatively different than in the undulator case.

In the following sections we undertake a comparison of the collective beam dynamics induced by the beamradiation interaction in an FEL and a magnetic chicane. For sake of simplicity we restrict ourselves to 1D models of this interaction. Because the FEL theory [8] is well understood, and because we will frequently refer to aspects of the FEL model, we begin our discussion with a brief summary of its basic results. In addition, there has recently been a tremendous improvement in understanding the model of the microbunching instability (CSR instability) in a bunch compressor $[9,10]$. Because this theory is rather complex, we present here a simplified, low-gain model of the instability, based on a few, but well-justified, assumptions. We identify first many points of comparison and contrast between the FEL and CSR instabilities. We then extend the model of CSR instability to the high-gain limit, noting similarities in the theoretical analysis and underlying physics of both CSR and FEL cases.

\section{MICROBUNCH INSTABILITY IN A FEL}

Free-electron lasers employ the collective instability of an electron bunch in an undulator to increase the electromagnetic radiation output power level orders of magnitude beyond the level of spontaneous radiation. In addition, this radiation is at least partly coherent, and so the system has characteristics of more conventional lasers. We now review these characteristics through a discussion of the basic theory of the FEL.
The analytical framework for FEL theory is well understood and we present here only the results relevant to our discussion. Three dimensional effects [11] such as diffraction are ignored. A 1D treatment implies among other characteristics that the radiation does not, as in the case of the chicane system, leave the region of the beam transversely, either by diffraction, or cross propagation. As we consider a long beam limit only, the radiation also does not leave the beam region longitudinally.

The FEL process is initiated by a preexisting modulation in the electron bunch distribution at the undulator fundamental resonance wavelength (self-amplified spontaneous emission free-electron laser, or SASE FEL), which may exist initially only at the random noise level. For a planar undulator the velocity of the electron in an alternating $B$ field $B_{y}(s)=B_{0} \sin \left(k_{U} s\right)$ of period $\lambda_{U}=$ $2 \pi / k_{U}$ and longitudinal position $s$ is in the $x z$ plane with

$$
\begin{aligned}
& \beta_{x}(s)=\frac{K}{\gamma} \sin \left(k_{U} s\right), \quad \beta_{y}(s)=0, \\
& \beta_{z}(s)=1-\frac{1+K^{2} / 2}{2 \gamma^{2}}+\frac{K^{2}}{4 \gamma^{2}} \cos \left(2 k_{U} s\right) .
\end{aligned}
$$

The undulator parameter $K$ is defined as $K=e B_{0} / m c k_{U}$. Because the electrons have a transverse velocity component, they couple with a superimposed radiation field, propagating along with the beam.

The change in the energy depends on the phase between the motion and the electromagnetic field $E_{x}(s, t)=$ $E_{0} \cos [k(s-c t)+\phi]$. The FEL model assumes that at any phase the interaction over a single period is too weak to exhibit significant effects. As the energy transfer has to be accumulated over many periods, it requires that the phase remains almost constant over many undulator periods. One frequency fulfills this requirement, yielding the FEL resonance condition

$$
\left\langle\beta_{z}\right\rangle=\frac{k}{k+k_{U}} .
$$

This condition physically implies that the radiation slips one wavelength past the beam electrons per undulator period, as it must to allow optimal constructive interference in the emitted radiation. The restriction of examining only a small bandwidth [12] around the resonant frequency which obeys Eq. (1) is referred to as the resonance approximation.

It significantly simplifies the analytical framework of FEL theory if the equations of motion are averaged over the undulator period. The longitudinal variables of each electron-the ponderomotive phase $\theta_{j}=\left(k+k_{U}\right) s_{j}-$ ckt and the energy deviation $\delta_{j}=\left(\gamma_{j}-\gamma_{0}\right) / \rho \gamma_{0}$ from the mean energy $\gamma_{0}$-are slow-varying quantities in the comoving frame of the ponderomotive wave. The scaling parameter [2] $\rho$ is introduced to simplify the equations of motion. It is defined as 


$$
\rho=\left[\frac{K f_{c} \gamma_{0}^{2} \omega_{p}}{\sqrt{32} c \gamma_{R}^{2} k_{U}}\right]^{2 / 3}
$$

where $\omega_{p}=\sqrt{\mu_{0} n_{e} e^{2} c^{2} / m \gamma_{0}^{3}}$ is the plasma frequency and $\gamma_{R}$ the resonant energy, which fulfills the requirement of Eq. (1) for a given radiation and undulator wave number. In a planar undulator the electrons also perform a longitudinal oscillation (when the transverse velocity is large, the longitudinal velocity is diminished) which effectively smears out the particle position. This reduces the coupling and is expressed by the coupling factor $f_{c}=$ $J_{0}(\eta)-J_{1}(\eta)$ with $\eta=K^{2} /\left(4+2 K^{2}\right)$. With the normalized radiation field amplitude $A=-i\left[K f_{c} /(2+\right.$ $\left.\left.K^{2}\right) \rho^{2}\right]\left(e E_{0} / m c^{2} k\right) e^{i \phi}$ the dynamics of an FEL is described by the FEL equations

$$
\begin{gathered}
\frac{d \theta_{j}}{d \hat{s}}=\Delta+\delta_{j}, \\
\frac{d \delta_{j}}{d \hat{s}}=-\left[\left(A+i \sigma\left\langle e^{-i \theta j}\right\rangle\right) e^{i \theta_{j}}+\text { c.c. }\right] \\
\frac{d A}{d \hat{s}}=\left\langle e^{-i \theta_{j}}\right\rangle .
\end{gathered}
$$

The detuning parameter $\Delta=\left(\gamma_{0}^{2}-\gamma_{R}^{2}\right) / 2 \rho \gamma_{R}^{2}$ is the collective deviation of the beam energy from the resonant energy. Note that the change in longitudinal position $\theta$ is linear with the total momentum offset $\Delta+\delta$. Thus the longitudinal velocity responds without delay to changes in momentum. Note that this is true only because the equations of motion are averaged over a period-examinations of delay in response shorter than one period are outside of the model's applicability.

We complete our description by noting that the space charge parameter $\sigma=\left(\omega_{p}^{2} \gamma_{0}^{2}\right) / 2 c^{2} k k_{U} \gamma_{R}^{2}$ represents the longitudinal repulsive forces which counteract the induced bunching by the radiation field. The normalized position $\hat{s}=2 c k_{u} \rho t$ is the position in the undulator, measured in units of the characteristic length $1 / 2 k_{u} \rho$.

Equations (3)-(5) are coupled and allow collective instability to occur. Assuming that the modulation in energy and position as well as the radiation field amplitude evolves exponentially as exp $[i \Lambda \hat{s}]$ the FEL equations are reduced to the dispersion relation [13]

$$
1+\left(\frac{1}{\Lambda}-\sigma\right) \int \frac{\partial f_{0}}{\partial \delta} \frac{1}{\Lambda+\Delta+\delta} d \delta=0,
$$

where $f_{0}$ is the initial distribution in energy. In the case of no detuning, space charge, and energy spread, the dispersion relation reduces to the cubic equation $\Lambda^{3}=-1$. One of the roots has an imaginary part of amplitude $-\sqrt{3} / 2$ which corresponds in an exponential growth of the field amplitude and the beam modulation. The characteristic scale of the field growth is the gain length $L_{g}=$ $\lambda_{U} / \sqrt{12} \pi \rho$.
A limit is implicitly imposed on the FEL equations by the resonance approximation. It can be expressed using the FEL parameter $\rho$ as

$$
\rho \ll 1
$$

For all existing and planned FELs [14-19] the condition is sufficiently fulfilled. However, the conceptual consequences of violating the condition in Eq. (7) will be explored in the context of our comparison of the FEL and CSR instabilities.

In summary, the FEL instability has several unique features, which we point out here to aid in our comparison with the CSR microbunching instability in a magnetic chicane:

Frequency components are located within a narrow bandwidth around a central, resonant wavelength Eq. (1). The relative bandwidth is of the order of $\rho$. If $\rho$ were to attain values near unity, the resonance approximation would clearly be violated, and the physical picture we have deduced from the analysis above would qualitatively change.

The amplitude of the transverse oscillation is typically small compared to the beam size. The same radiation field interacts with the electron bunch over the entire undulator length. This requires one to include Maxwell equations for a self-consistent description of the instability process. Assuming validity of the resonance approximation and the resulting predominant direction of propagation of the radiation field at a well-defined wavelength implies the paraxial approximation of the Maxwell equations, where the second order derivatives of the radiation amplitude and phase with respect to longitudinal position and time are ignored.

The electron dynamics occurring during an undulator period is replaced by its averaged behavior. As with the paraxial approximation of the radiation field, this is valid as long as the resonance approximation holds. Again, if $\rho$ is near unity, the paraxial approximation (and the related slowly varying field amplitude approximation) would be violated, and the standard description of the FEL we have given is not valid.

\section{DESCRIPTION OF PARTICLE DYNAMICS IN A MAGNETIC CHICANE}

A magnetic chicane consists typically of bending magnets, drifts, and, optionally, focusing quadrupoles. For a simpler comparison to the FEL we exclude the latter two components from the discussion. The bend angle in a chicane dipole depends on the beam energy, and the orbit within the chicane is dispersive. If the electron energy is constant the path length difference in the bunch compressor can be expressed by the matrix element $R_{56}=$ $\left[\partial \zeta(s) / \partial \delta\left(s_{0}\right)\right]$, with

$$
\zeta(s)=\zeta\left(s_{0}\right)+R_{56}\left(s, s_{0}\right) \delta .
$$


Here $s$ and $s_{0}$ denote the final and initial positions on the design orbit, respectively, $\zeta=c t$ is the position in the frame of the moving bunch, and $\delta=\left(\gamma-\gamma_{0}\right) / \gamma_{0}$ is the normalized deviation of the electron energy from the mean energy $\gamma_{0}$; we are using the ultrarelativistic approximation $\beta=1$. The matrix element $R_{56}$ depends on the dispersion function $\eta$ and the bend radius $R$ and is defined as

$$
R_{56}\left(s, s_{0}\right)=\int_{s_{0}}^{s} \frac{\eta\left(s^{\prime}\right)}{R\left(s^{\prime}\right)} d s^{\prime}
$$

For the case where the electron beam interacts with the spontaneous radiation, the dispersion functions change with any change in the electron energy. We thus generalize the definition of $R_{56}$ to

$$
\zeta(s)=\zeta\left(s_{0}\right)+\int_{s_{0}}^{s} \delta\left(s^{\prime}\right) \tilde{R}_{56}\left(s, s^{\prime}\right) d s^{\prime}
$$

In the case that $\delta$ is constant Eq. (10) reduces to Eq. (8) using the relation $R_{56}\left(s, s_{0}\right)=\int_{s_{0}}^{s} \tilde{R}_{56}\left(s, s^{\prime}\right) d s^{\prime}$. The interpretation of Eq. (10) is that the change in $\zeta$ is given by the summation over all dispersion contributions between two arbitrary points $s$ and $s_{0}$, wherever the beam energy changes. The longitudinal "velocity" is easily obtained by taking the derivative of Eq. (10), yielding

$$
\frac{d \zeta}{d s}=\int_{s_{0}}^{s} \delta\left(s^{\prime}\right) \frac{\partial \tilde{R}_{56}\left(s, s^{\prime}\right)}{\partial s} d s^{\prime} .
$$

Note that the derivative with respect to the upper boundary of the integral of Eq. (10) is zero. The function $\tilde{R}_{56}$ implicitly depends on the bend field and radiated energy at all preceding positions in the magnetic chicane.

To describe a microbunched distribution of electrons, we begin by assuming a coasting beam with a small modulation

$$
I(\zeta, s)=I_{0}\{1+|b(s)| \cos [k \zeta+\phi(s)]\} .
$$

Because the magnitude and the relative position of the modulation can change within the bunch compressor, $|b|$ and $\phi$ depend on $s$. The emission of synchrotron radiation interacts with electrons in the forward direction. The resulting potential [6] seen by the electrons is

$$
W(\zeta)=-\frac{2}{\left(3 R^{2}\right)^{1 / 3}} \frac{1}{\zeta^{1 / 3}} \frac{\partial}{\partial \zeta}
$$

for $\zeta<0$ and zero otherwise. Note that in this model we exclude any transient effects and assume that the interaction is instantaneous. The energy change of any given electron is

$$
\begin{aligned}
\frac{d \delta}{d s} & =-\frac{I_{0}}{I_{A} \gamma_{0}} \int_{-\infty}^{\infty} W\left(\zeta-\zeta^{\prime}\right) I\left(\zeta^{\prime}\right) d \zeta^{\prime} \\
& =-\frac{I_{0}}{I_{A} \gamma_{0}} \frac{2 k|b(s)|}{\left(3 R^{2}\right)^{1 / 3}} \int_{\zeta}^{\infty} \frac{\sin \left[k \zeta^{\prime}+\phi(s)\right]}{\left(\zeta^{\prime}-\zeta\right)^{1 / 3}} d \zeta^{\prime} \\
& =-\frac{I_{0}}{I_{A} \gamma_{0}} \frac{2 \Gamma\left(\frac{2}{3}\right) k^{1 / 3}}{\left(3 R^{2}\right)^{1 / 3}}|b(s)| \sin \left(k \zeta+\phi(s)+\frac{\pi}{3}\right)
\end{aligned}
$$

The growth of the energy modulation scales linearly with the modulation of the current. Note also that the potential has a phase offset of $-\pi / 6$ with respect to the modulation itself.

\section{THE CHICANE MODEL}

To perform a first analysis of the CSR instability, we consider an idealized chicane, which consists of three bending magnets with no drift space separating them. The outer magnets have a length of $L$ while the inner one, which bends in the opposing direction of the outer two, is twice as long. This model, which describes many existing chicanes well, may not apply to certain proposed chicanes, which have long intermagnet drifts.

To further simplify our calculations, the bend radius $R$ is assumed to be much larger than $L$ and the dispersion function is calculated based on the small deflection angle approximation $\theta_{b} \approx L / R \ll 1$. It is convenient to normalize the dispersion function $\eta$ between any two arbitrarily chosen positions $s$ and $s^{\prime}$ to the bend radius $R$. With the initial conditions $\eta\left(s^{\prime}\right)=0$ and $\eta^{\prime}\left(s^{\prime}\right)=0$ the dispersion function is

$$
\frac{\eta}{R}= \begin{cases}\frac{1}{2}\left(\frac{s-s^{\prime}}{R}\right)^{2} & (\text { I, I }),(I I, I I), \\ \left(\frac{s-L}{R}\right)^{2}-\frac{1}{2}\left(\frac{s-s^{\prime}}{R}\right)^{2} & \text { (III, III), } \\ \left(\frac{s-3 L}{R}\right)^{2}-\frac{1}{2}\left(\frac{s-s^{\prime}}{R}\right)^{2} & (\text { II, I), } \\ \frac{1}{2}\left(\frac{s-3 L}{R}\right)^{2}+\frac{1}{2}\left(\frac{3 L-s^{\prime}}{R}\right)^{2}-\left(\frac{2 L}{R}\right)^{2} & (\text { III, II), } \\ -\left(\frac{s-3 L}{R}\right)\left(\frac{L-s^{\prime}}{R}\right) & (\text { III, I), }\end{cases}
$$

where the pairs of roman numbers indicate in which dipole the ending and starting points, respectively, are located. The dispersion function is related to the modified matrix element $\tilde{R}_{56}$ as

$$
\frac{\partial}{\partial s} \tilde{R}_{56}=-\frac{\partial}{\partial s^{\prime}} \frac{\eta}{R}=\frac{s-s^{\prime}}{R(s) R\left(s^{\prime}\right)} .
$$

We incorporated the bend direction of the dipoles into the sign of the bend radius as

$$
R(s)= \begin{cases}R, & s \text { in } 1 \text { st or 3rd dipole } \\ -R, & s \text { in } 2 \text { nd dipole }\end{cases}
$$

With this convention, it can be easily seen that the sign of the differential matrix element changes when the electron enters the adjacent dipole. Physically this means that a particle that gains energy, becoming more rigid, has the 
tendency to fall behind the design trajectory in the magnet where the energy gain occurs. At a magnet boundary, however, the change in the dipole polarity reverses this effect, and the higher energy particle moves forward with respect to the design trajectory. In a chicane where the energy is held constant, the beam actually decompresses in the first and last dipoles, and compresses in the middle. Net compression is achieved in this case because the dispersion is much larger in the middle dipole.

In the present model, the differential equation for the longitudinal motion becomes

$$
\frac{d \zeta}{d s}=\int_{0}^{s} \delta\left(s^{\prime}\right) \frac{s-s^{\prime}}{R(s) R\left(s^{\prime}\right)} d s^{\prime} .
$$

\section{THE LOW-GAIN MODEL}

As indicated in Eq. (18) a change in the particle energy has a delayed effect on the particle's longitudinal position, which grows with the third power in $s$. In addition the change in the longitudinal position is also inhibited by the change in the polarity of the bending magnets. Particles with higher energy fall behind due to the larger bend radius, but catch up due to the shorter path length after a polarity change. Similar arguments are valid for lower energy particles. A third condition must be present before longitudinal motion and therefore instability can grow-the energy modulation must first grow significantly. Thus for short time scales, and low gain, $\delta\left(s^{\prime}\right)$ can be expected to change linearly in $s^{\prime}$.

We recall for comparison that the resonance condition of the FEL theory averages over one period, and as a result the change in the longitudinal position due to an energy modulation is linear in $s$. Therefore the differential equations for phase and energy must be solved simultaneously. For the chicane, assuming low gain, the situation is qualitatively different. We can expect that the energy modulation is accumulated first before it affects the longitudinal position, which changes primarily in the last part of the chicane. The mechanism is very similar to that of a klystron [20].

This assumption of klystronlike behavior is invalid for high currents. In this case the delayed change in the longitudinal position is noticeable even in the first bending magnet. This instability is similar to the bunching instability in a storage ring [21], which can be regarded as a single long bending magnet. An initial bunching will be amplified exponentially until all particles are bunched and the beam breaks up. We discuss this limit further in the following section.

For many of the presently operating or proposed chicane compressors, the low-gain limit is valid. We model the initial current by an equidistant distribution plus an added sinusoidal modulation in the positions. The amplitude and wave number of this modulation are $\Delta \zeta$ and $k$.
The complex-value bunching factor is determined by

$$
\begin{aligned}
b= & \frac{1}{N} \sum_{j=1}^{N} \exp \left[-i k \zeta_{j}+i k \Delta \zeta \sin \left(k \zeta_{j}+\phi\right)\right] \\
& \frac{1}{N} \sum_{j=1}^{N} \exp \left[-i k \zeta_{j}\right]\left[\sum_{n=-\infty}^{\infty} J_{n}(k \Delta \zeta) \exp \left[i n\left(k \zeta_{j}+\phi\right)\right]\right] \\
& =J_{1}(k \Delta \zeta) e^{i \phi} .
\end{aligned}
$$

Because the effective radiation potential is harmonic in $\zeta$ the resulting modulation in the longitudinal position is harmonic as well with $\delta \zeta=Z(s) \sin \left(k \zeta_{0}+\psi\right)$, where $\zeta_{0}$ is the initial position of a particle and $Z(s)$ vanishes at the entrance of the chicane. The resulting bunching factor becomes

$$
b(s)=\sum_{n+m=1} J_{n}(k \Delta \zeta) J_{m}(k Z(s)) e^{i n \phi+i m \psi} .
$$

In our low-gain model the initial offset $\Delta \zeta$ and the modulation amplitude $Z(s)$ are much smaller than the modulation wavelength. This implies that in the Taylor series expansion of the Bessel functions in Eq. (20) the lowest order term is linear either in $\Delta \zeta$ or $Z(s)$. Further the initial amplitude of the longitudinal modulation is small compared to the modulation wavelength. As long as $Z(s)$ is comparable to the initial modulation amplitude $\Delta \zeta$, the bunching factor can be taken as constant and Eq. (14) can easily be integrated, giving a linear dependence on $s$. Inserting $\delta(s)$ into Eq. (18), the longitudinal position evolves in the chicane as

$$
\begin{aligned}
\zeta_{j}(s)= & \zeta_{j}(0)-\frac{I_{0}}{I_{A} \gamma_{0}} \frac{2 \Gamma\left(\frac{2}{3}\right) k^{1 / 3}}{\left(3 R^{2}\right)^{1 / 3}}|b(0)| \\
& \cdot \sin \left(k \zeta_{j}(0)+\phi+\frac{\pi}{3}\right) \Phi(s),
\end{aligned}
$$

with

$$
\begin{aligned}
\Phi(s) & =\int_{0}^{s}\left[\int_{0}^{s^{\prime \prime}} \frac{s^{\prime}\left(s^{\prime \prime}-s^{\prime}\right)}{R\left(s^{\prime \prime}\right) R\left(s^{\prime}\right)} d s^{\prime}\right] d s^{\prime \prime} \\
& = \begin{cases}\frac{1}{24} \frac{s^{4}}{R^{2}} & \text { (I), } \\
\frac{1}{24} \frac{s^{4}}{R^{2}}-\frac{1}{2} \frac{L^{2}}{R^{2}} s^{2}+\frac{2}{3} \frac{L^{3}}{R^{2}} s-\frac{1}{6} \frac{L^{4}}{R^{2}} & \text { (II), } \\
\frac{1}{24} \frac{s^{4}}{R^{2}}-4 \frac{L^{2}}{R^{2}} s^{2}+\frac{52}{3} \frac{L^{3}}{R^{2}} s-\frac{56}{3} \frac{L^{4}}{R^{2}} & \text { (III). }\end{cases}
\end{aligned}
$$

The roman numerals indicate the dipole in which the position $s$ lies. Figure 1 shows the universal function $\Phi(s)$. The function can be characterized differently in four sections. In section 1 (first dipole, I) an electron, which gains energy, is slowed down because the bend radius and thus the path length gets larger. Section 2 is the first half of the second dipole (II), where the longitudinal motion is inverted as the polarity of the magnetic field changes. In the second half of the second dipole the "slow-down" effect of the increasing bend radius takes over. Finally in 


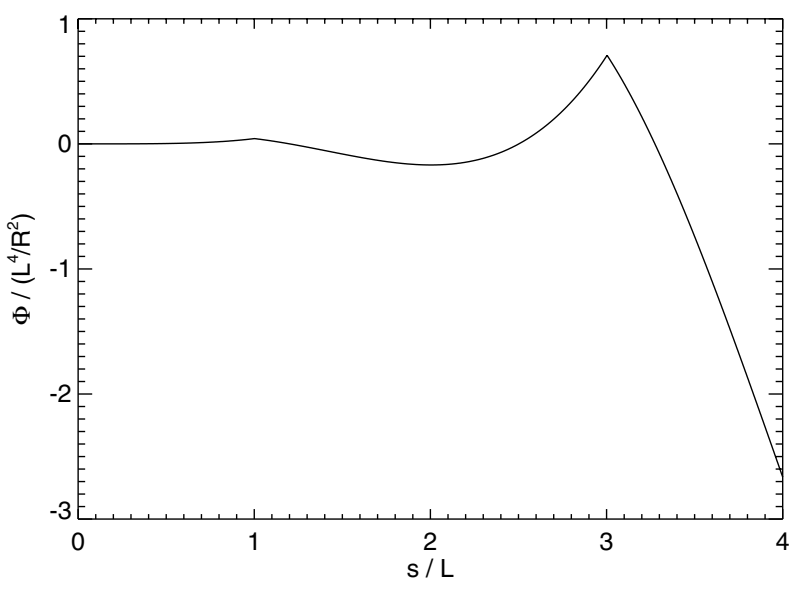

FIG. 1. The function $\Phi$ [Eq. (22)].

the third and last magnet (section 4, dipole III) the path length changes originating in the first and second dipoles adding up with the same sign. Here the strongest change in the longitudinal position occurs. Somewhat surprisingly, the final offset in $\zeta$ is identical to that calculated if the accumulated energy change is applied directly at the entrance of the chicane and the electron is tracked by using the standard expression for the matrix element $R_{56}$.

The value of $\Phi$ at the end of the chicane is $-(8 / 3)\left(L^{4} / R^{2}\right)$, which also defines the maximum offset in the longitudinal direction a particle will see. The final, normalized amplitude is

$$
\xi=\frac{I_{0} \Gamma\left(\frac{2}{3}\right)}{2 I_{A} \gamma_{0}}\left(\frac{8 L^{3} k}{3 R^{2}}\right)^{4 / 3} .
$$

With $k Z(4 L)=\xi|b(0)| \ll 1$ the gain, defined as the ratio between final and initial amplitude of modulation, becomes

$$
G=\frac{|b(4 L)|}{|b(0)|}=\sqrt{1+\xi+\xi^{2}}
$$

or $G \approx \xi$ in the limit of large values for $\xi$. Expressed in terms of the $R_{56}$ matrix element it becomes

$$
G \approx \frac{I_{0} \Gamma\left(\frac{2}{3}\right)}{2 I_{A} \gamma_{0}}\left(2 k R_{56}\right)^{4 / 3}
$$

which is in agreement with the results in Ref. [9], although a different model for the bunch compressor has been used. Note that the gain is broadband, not resonant. This stands in contrast to the FEL instability, where resonant behavior is derived from the periodic nature of the beam-undulator-radiation interaction.

As an example of a generic magnetic chicane, modeling the first Linac Coherent Light Source (LCLS) bunch compressor $\left(\gamma=500, I_{0}=100 \mathrm{~A}, R=12 \mathrm{~m}\right.$, and $L=$ $1.5 \mathrm{~m}$ ), an initial modulation with a period of $5 \mu \mathrm{m}$ would grow by a factor of 25 . The notion of "low gain" is somehow misleading in this context. We use this ex- pression because the gain scales roughly linearly with the beam current. This regime of operation is named "onestage amplification" [9]. This is identical with an optical klystron, a low-gain FEL [4], where the gain is enhanced by a dispersive section. In the FEL high-gain regime, as discussed in Sec. II, the dependence is exponential. We will also find similar exponential dependence in the highgain regime of the CSR instability.

\section{ENERGY SPREAD AND COMPRESSION}

The gain growth, discussed above, has a singularity at a zero modulation period length. This artifact is removed if energy spread is included in the model. With finite uncorrelated (thermal) energy spread, any modulation in the beam current is spread out and the emission level of the synchrotron radiation is reduced. This effect is particularly strong at short modulation wavelengths. Thus one expects that there is an optimum wavelength for gain of the CSR-induced modulation, where the natural tendency of the system to amplify shorter wavelengths is not yet damped by thermal effects. This type of instability suppression is often designated as Landau damping.

We now provide an analysis of energy spread effects. Including energy spread, the current profiles evolve as

$$
I=I_{0}\left[1+\int f(\delta)|b(0)| \cos \left[k\left(\zeta+R_{56} \delta\right)+\phi\right] d \delta\right],
$$

where $f(\delta)$ is the normalized energy distribution. We assume no correlation between energy and longitudinal position on the scale of the modulation. For a Gaussian energy distribution with rms spread $\sigma_{\delta}$ the integration of this equation yields

$$
I=I_{0}\left[1+e^{-(1 / 2)\left(\sigma_{\delta} R_{56} k\right)^{2}}|b(0)| \cos (k \zeta+\phi)\right] .
$$

The modulation decays if the spread in the longitudinal position $\sigma_{\delta} R_{56}$ is comparable with the modulation period length. The initial modulation is sheared mainly in the second dipole, where the value of matrix element $R_{56}$ changes significantly. Because the seed for the microbunch instability - the accumulated change in the electron energy-occurs before that, the change in the longitudinal position [Eq. (22)] is hardly affected. We can just apply the damping factor due to the energy spread to the previous results of Eq. (24). With $R_{56}(4 L, 0)=$ $-(4 / 3) L^{3} / R^{2}$ the final gain is

$$
G=e^{-\alpha|\xi|^{3 / 2}} \sqrt{1+\xi+\xi^{2}},
$$

defining the normalized energy spread as

$$
\alpha=\sqrt{\left(\frac{I_{A} \gamma_{0}}{2 I_{0} \Gamma\left(\frac{2}{3}\right)}\right)^{3}} \sigma_{\delta}^{2} .
$$

For the LCLS case with an energy spread of $0.01 \%$ $(\alpha=0.05)$, the gain at $5 \mu \mathrm{m}$ would be reduced by 7 orders 


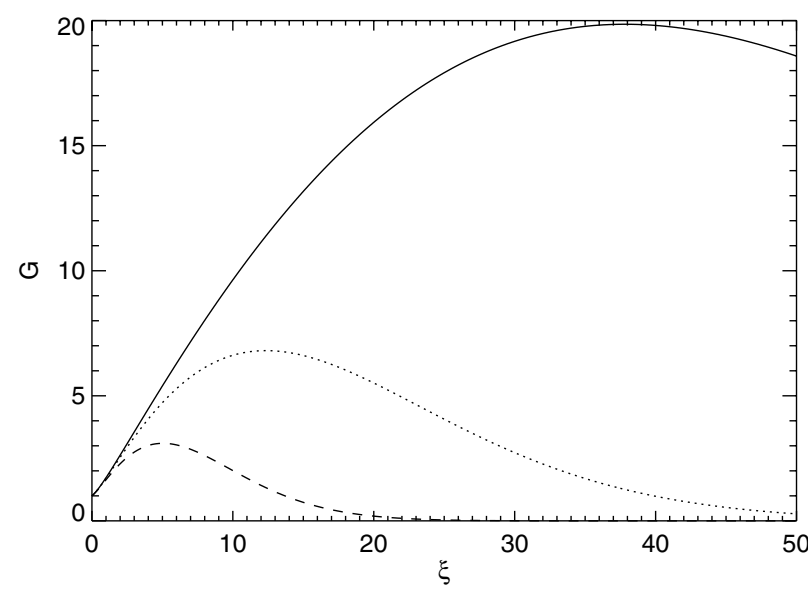

FIG. 2. Gain curve for energy spreads of $\alpha=0.003,0.015$, and 0.05 (solid, dotted, and dashed lines, respectively).

of magnitude. Figure 2 shows the achievable gains for different energy spreads.

In the case that a linear chirp is imposed on the beam to compress it, the path length differences in the chicane change the period length of the modulation. With the compression factor

$$
C(s)=\frac{1}{1+h R_{56}(s, 0)}
$$

and the chirp gradient $h=d \delta / c d t$, the wave number $k$ scales along the chicane as $k(s)=C(s) k(0)$. This results in a larger wakefield amplitude although the dependence is rather weak due to the cubic root of the wave number in Eq. (14). Further, this wakefield enhancement is mainly visible in the last dipole. Because of the same reason the increase of the current due to compression has only limited impact on the gain of the microbunch instability. As in our discussion of the energy spread, the induced energy modulation in the first half of the chicane drives

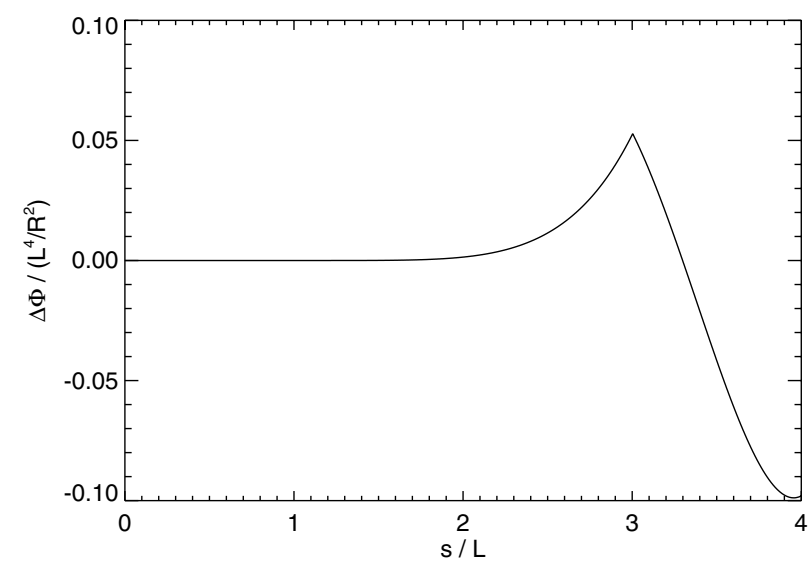

FIG. 3. Change in the longitudinal orbit $\Phi$ due to a compression by a factor of 10 . the longitudinal motion. Figure 3 shows the change in the function $\Phi$ for a strong compression by a factor of 10 , solving Eqs. (14) and (18) self-consistently. The maximum change is less than $10 \%$. The remaining effect of compression is that the electrons move farther with respect to the reduced modulation wavelength. The normalized amplitude and energy spread scales as

$$
\xi \rightarrow C \xi \quad \text { and } \quad \alpha \rightarrow \sqrt{C} \alpha
$$

\section{THE HIGH-GAIN MODEL}

In our low-gain model we assumed that the CSR microbunching instability does not drive any bunching within the first dipole. To qualitatively place a limit on this assumed scenario, we develop here a high-gain, exponential growth model as well. There may be situations where the exponential gain does not assert itself in the first dipole, but may, by compression and thus higher current, become notable in the last dipole. We shall see that the exponentially growing regime of the CSR instability has many similarities with the high-gain FEL.

To begin our analysis, we define the collective variables

$$
\begin{gathered}
B=-i k\left\langle e^{-i \Psi} \zeta\right\rangle, \\
\Delta=\left\langle e^{-i \Psi} \delta\right\rangle,
\end{gathered}
$$

where $\Psi_{j}=k \zeta_{0, j}$ is the initial phase of the $j$ th electron of a uniform distribution. Note that $B$ is the Taylor series expansion of the bunching factor $b$ [Eq. (20)]. The equations of motion for a cold beam become

$$
\begin{gathered}
\frac{d \Delta}{d s}=-\frac{\rho_{\mathrm{CSR}}^{4}}{k R^{2}} e^{i(\pi / 3)} B, \\
\frac{d B}{d s}=-i \frac{k}{R^{2}} \int_{0}^{s} \Delta\left(s-s^{\prime}\right) d s^{\prime},
\end{gathered}
$$

with the definition (in direct analogy with the FEL case) of the dimensionless $\rho_{\mathrm{CSR}}$ parameter

$$
\rho_{\mathrm{CSR}}=\left[\frac{I_{0}}{I_{A} \gamma_{0}} 4^{1 / 3} \Gamma\left(\frac{2}{3}\right)\right]^{1 / 4}(k R)^{1 / 3} .
$$

The equations can be combined into a fourth order differential equation. Using the ansatz $B \propto \exp [i \Lambda s]$ we obtain the dispersion relation

$$
\Lambda^{4}=\left(\frac{\rho_{\mathrm{CSR}}}{R}\right)^{4} e^{i(5 \pi / 6)} .
$$

Four solutions exist, where two of the roots have a negative imaginary part, corresponding to an exponentially growing instability. The growth rates are $\left(\rho_{\mathrm{CSR}} / R\right) \sin (7 \pi / 24)$ and $\left(\rho_{\mathrm{CSR}} / R\right) \sin (5 \pi / 24)$, respectively. An estimate for the growth rate has been derived in [20], which differs only by a few percent from the exact 
solution, given above. Just as the gain length of the high-gain FEL scales as $\lambda_{U} / \rho$, the gain length of the high-gain CSR instability is roughly $R / \rho_{\text {CSR }}$. Because the calculations are based on a relatively small deflection angle $(L \ll R)$ exponential gain within a single dipole becomes significant only for

$$
\rho_{\mathrm{CSR}}>\frac{R}{L} \gg 1
$$

In comparison to the FEL the dispersion relation for CSR instability is of fourth order, because the change in the bunching factor is of third order in energy, while the energy modulation is linear in the bunching. For the FEL model, all equations (energy, phase, and radiation field) are linear, resulting in a third order expression.

The startup regime of the high-gain CSR instability is of particular interest because it determines after how many gain lengths the exponential growth becomes dominant. In the case of an FEL this is typically achieved after the first or second gain length. Here the situation is different because the number of modes is higher. In addition the two growing modes have similar growth rates but different phase slippages (real part of $\Lambda$ ), so that the interference between these two is still noticeable after several gain lengths. Figure 4 shows the growth rate normalized to $\left|\operatorname{Im}\left(\Lambda_{1}\right)\right|=\left(\rho_{\mathrm{CSR}} / R\right) \sin (7 \pi / 24)$. It takes at least five gain lengths before the bunching factor has grown by 1 order of magnitude, compensating an initial amplitude drop. The interference with the other growing mode is still notable after 20 gain lengths.

The integral in Eq. (34) prevents an easy solution of the Vaslov equation to include energy spread effects. We conclude this section with a qualitative analysis. For a characteristic spread $\sigma_{\delta}$ in energy the momentum dispersion couples it to a phase spread of approximately $\left(k / 6 R^{2}\right) \sigma_{\delta} s^{3}$. In units of the gain length $\left(\hat{s}=s \rho_{\mathrm{CSR}} / R\right)$

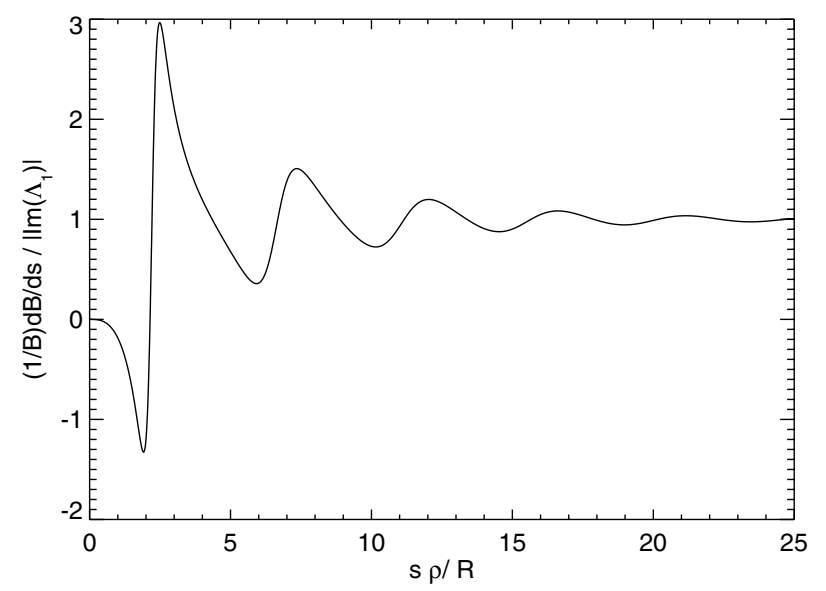

FIG. 4. Growth rate of the bunching factor, normalized to the growth rate of the dominant growing mode. the normalized phase spread $\hat{\sigma}_{\delta}=\left(k R / 6 \rho_{\mathrm{CSR}}^{3}\right) \sigma_{\delta}$ is independent of the bend radius and wavelength. An estimate on the mitigation of the instability by the energy spread can be obtained by the condition that for a Gaussian-spectrum beam the growth over five gain lengths is canceled by the phase spread. The threshold for the normalized energy spread is $\hat{\sigma}_{\delta}<0.02$ by this criterion - any value larger than 0.02 would completely suppress the exponential growth of this instability. Note that if even the constraint is fulfilled the energy spread may affect the instability at a certain point due to its strong dependence on $s$. For a Gaussian spread this occurs after $\hat{\sigma}_{\delta}^{-1 / 3}$ gain lengths. Because the gain length depends on $k$, longer wavelengths are less affected by the energy spread, as we have seen in the low-gain analysis already.

\section{CONCLUSIONS}

Because of the mechanism of coherent synchrotron radiation-induced energy feedback, any transverse deflecting beam system may support a microbunching instability. The underlying dynamics of these instabilities may be quite different, however. In the cases we have considered, the undulator and the magnetic chicane, these differences are particularly strong. In an FEL the local interaction is rather weak over one period, and must be accumulated over many bending oscillations to yield an instability. It is quite important to the FEL instability that the emitted radiation stays in the electron beam region, therefore, to allow a significant buildup of the beamradiation interaction. The relative weakness of this interaction is expressed by the value of the $\rho$ parameter, which is much smaller than unity, indicating a slowly varying system.

Theoretically, an important measure of the transition between the FEL instability and the microbunching instability in a chicane would be the extrapolation of $\rho$ to unity and beyond. It should be emphasized that the use of the standard analytical model in this case would not yield reasonable results, as the assumptions (slowly varying system, paraxial radiation) would be violated. A valid theory in this limit must include the entire radiation field in all directions, as well as the explicit longitudinal motion variations of the electrons. Some of these characteristics are present in our chicane-based analysis, which is not fully general. In a chicane, the orbit offset is large compared to the beam size so that an emitted radiation field interacts only briefly with the electrons before escaping the beam. Under this assumption the radiation field dynamics have been simplified to a wake potential which acts instantaneously on the beam. A general treatment, covering both FEL-like and chicanelike cases, would demand a much more sophisticated model of the radiation emission. 


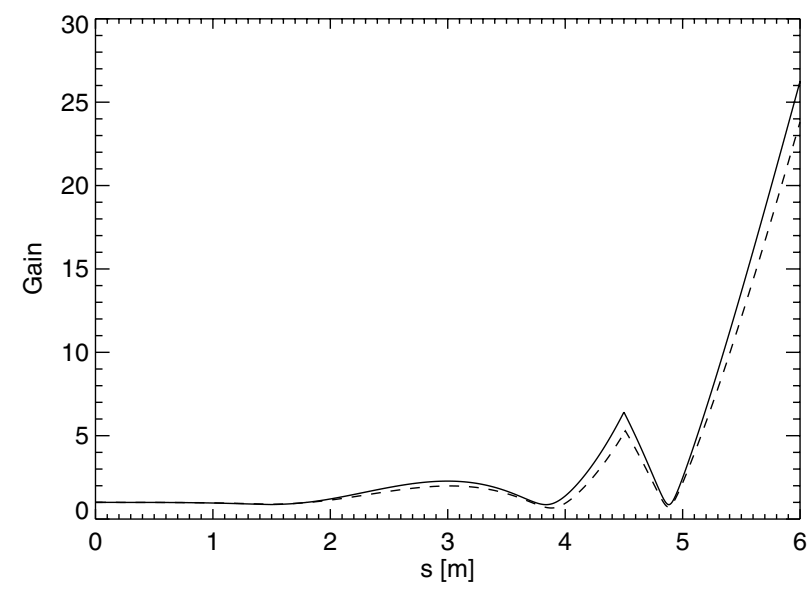

FIG. 5. Evolution of the gain along the chicane, using the low-gain model [Eq. (24)] and a self-consistent numerical simulation (solid and dashed lines, respectively). The input parameters are $I_{0}=100 \mathrm{~A}, \gamma_{0}=500, R=12 \mathrm{~m}$, and $L=$ $1.5 \mathrm{~m}$.

We have derived a simple low-gain model to calculate the growth of an initial current modulation within a magnetic chicane. As in a klystron the physics is split into the initial modulation in beam energy, and a change in the longitudinal position (followed by an enhanced emission level of radiation). The major assumption in our low-gain model is that the beam current modulation, which generates the coherent synchrotron radiation, is held constant over the entire chicane. The klystronlike assumption implies that the system does not have an exponential gain, but rather acts as a linear amplifier where the gain coefficient is dependent on the physical details of the beam and chicane. This is in striking resemblance with the low-gain model of an FEL [22]. Figure 5 shows the difference, however, between our klystronlike model and a self-consistent, more complex model [10].

In order to extend the low-gain model to account for self-consistent behavior, we have developed a high-gain model that has notable similarities with high-gain FEL theory. In particular, we obtained a quartic dispersion relation which allows us to scale the gain through a $\rho$ parameter $\left(\rho_{\mathrm{CSR}}\right)$, with a gain length proportional to $R / \rho_{\mathrm{CSR}}$. In contrast to the FEL case, with the CSR instability, the parameter $\rho_{\mathrm{CSR}}$ must have a value in excess of unity, so that exponential gain occurs within a single dipole.

In conclusion, a microbunching instability, displaying familiar behaviors such as klystronlike or exponentially growing, occurs in transverse deflecting magnetic devices. Different assumptions and approximations allow this instability to be investigated analytically, within the confines of physically relevant models, such as the FEL and the compressor chicane. The FEL analysis relies on a simplified, period-averaged view of the electron motion, allowing an exponentially growing instability. In the case of the magnetic chicane, on the other hand, electron motion must be treated in a more intricate way, but the radiation field can be simply expressed by a continuous force acting to change the beam energy. The transition between FEL and the CSR instability is controlled by the strength of the $\rho$ parameter; when it nears unity, a CSRlike case would occur. As the $\rho$ parameter is a valid number only when it is small, it is really only of value for a FEL-like (resonance approximation, paraxial radiation) case. For the CSR instability, it is more valuable to define the $\rho_{\text {CSR }}$ parameter in the context of exponential growth in a single bend magnet. With these issues in mind, we can classify bending systems in a hierarchy of gain strength, from lowest to highest: (1) standard FEL instability, where $\rho \ll 1$; (2) ultrahigh gain FEL $\rho \simeq 1$, where the standard theory is no longer valid; (3) CSR instability in the low-gain limit, where $\rho_{\mathrm{CSR}}$ is not greater than 1 , and the system is klystronlike, requiring the entire chicane to develop; (4) high-gain CSR instability, where $\rho_{C S R}>1$ and the beam microbunches in one chicane magnet. At present, the only systems which are commonly encountered are (1) and (3). The relevance of analysis of systems (2) and (4) awaits further progress in high brightness electron beam production.

[1] J. Madey, J. Appl. Phys. 42, 1906 (1971).

[2] R. Bonifacio, C. Pellegrini, and L. M. Narducci, Opt. Commun. 50, 373 (1984).

[3] A. M. Kondratenko and E. L. Saldin, Part. Accel. 10, 1980 (1980).

[4] W. Colson, Phys. Rev. Lett. 36, 717 (1976).

[5] L. V. Iogansen and M. S. Rabinovich, Sov. Phys. JETP 37, 83 (1960).

[6] Y.S. Derbenev, J. Rossbach, E. L. Saldin, and V. D. Shiltsev, Deutsches Elektronen-Synchrotron Report No. TESLA-FEL 95-05, 1995.

[7] H. Braun, Phys. Rev. Lett. 84, 658 (2000).

[8] E. L. Saldin, E. A. Schneidmiller, and M.V. Yurkov, The Physics of Free Electron Lasers (Springer-Verlag, Berlin, 2000).

[9] Z. Huang and K.-J. Kim, in Proceedings of the 24th Advanced ICFA Beam Dynamics Workshop on Future Light Sources, Japan, 2002 (SPRING-8, Japan, 2002).

[10] S. Heifets, S. Krinsky, and G. Stupakov, Stanford Linear Accelerator Center Report No. SLAC-PUB9165, 2002.

[11] G. Moore, Opt. Commun. 52, 46 (1984).

[12] J. M. Wang and L.-H. Yu, Nucl. Instrum. Methods Phys. Res., Sect. A 250, 484 (1986).

[13] J. B. Murphy and C. Pellegrini, in Proceedings of the South Padre Island Conference, 1986 (Springer, New York, 1986).

[14] M. Hogan et al., Phys. Rev. Lett. 81, 4867 (1998).

[15] A. Tremaine et al., Nucl. Instrum. Methods Phys. Res., Sect. A 483, 24 (2002). 
[16] S. M. Milton et al., Science 292, 2037 (2001).

[17] V. Ayvazyan et al., Phys. Rev. Lett. 88, 104802 (2002).

[18] Linac Coherent Light Source (LCLS) Design Study Report No. SLAC-R-521 UC-414, 1998.

[19] TESLA Technical Design Report No. TESLA-FEL 2001-05, 2001.
[20] E. L. Saldin, E. A. Schneidmiller, and M.V. Yurkov, Deutsches Elektronen-Synchrotron Report No. TESLAFEL 2002-02, 2002.

[21] S. Heifets and G. Stupakov, Stanford Linear Accelerator Center Report No. SLAC-PUB-8761, 2001.

[22] W. B. Colson, Phys. Quantum Electron. 5, 157 (1978). 